\title{
Ocupação e hipertensão
}

\section{Work and high blood pressure}

\author{
Ricardo Cordeiro * , Frida M. Fischer ${ }^{\star \star}$,Euclydes C. Lima Filho ${ }^{\star \star \star}$,Djalma C. Moreira Filho ${ }^{\star \star \star *}$ \\ CORDEIRO, R. et al. Ocupação e hipertensão. Rev. Saúde Pública, 27:380-7, 1993. Com base em revisão \\ bibliográfica discute-se a literatura produzida nas décadas de 70 e 80 , no campo da epidemiologia da \\ hipertensão arterial sistêmica entre trabalhadores. Analisa-se não apenas o ponto de vista do conhecimento \\ gerado, mas também os aspectos relacionados ao instrumental térico-metodológico empregado.
}

Descritores: Hipertensão, epidemiologia. Ocupações.

\section{Introduçáo}

O grupo das doenças cardiovasculares tem sido apontado como a principal causa de óbitos no Brasil desde os anos 70 (FundaçãoIBGE ${ }^{27}, 1980$ ). Além disso, sua importância vem crescendo em relação às outras patologias. Em 1930 respondia por $11,8 \%$ dos óbitos no país, alcançando $25,8 \%$ em 1984 (Possas $^{84}, 1989$ ), sendo responsável naquela ocasião por cerca de $1 / 3$ dos бbitos nas regiões sul e sudeste (Ministério da Saúde ${ }^{71}$, 1987).

A hipertensão arterial sistêmica(HAS) como redutora da expectativa de vida é um fato bem estabelecido há mais de um século (Roccella e col $\left.{ }^{88}, 1987\right)$. Ela tem sido apontada como o fator de risco mais importante para as doenças cardiovasculares. Cerca de $80 \%$ das mortes por acidente vascular cerebral e $40 \%$ dos 6 bitos por doença coronariana seacompanham deHAS (Costa $\&$ Klein $\left.^{16}, 1985\right)$. Além disso, a doença hipertensiva éa responsável direta por cerca de 5\% dos óbitos dentro do grupo das doenças cardiovasculares.

Não é demais, ainda uma vez, repetir que, com relação às doenças cardiovasculares - e em particular à hipertensão arterial sistêmica - existem no Brasil alguns conceitos bastante difundidos, mas que não encontram

* Departamento de Saúde Pública da Faculdade de Medicina de Botucatu (UNESP) - Botucatu, SP - Brasil

** Departamento de Saúde Ambiental da Faculdade de Saúde Pública da Universidade de São Paulo - São Paulo, SP - Brasil

*** Departamento de Estatística do Instituto de Matemática, Estatística e Ciências da Computação da UNICAMP Campinas, SP - Brasil

**** Departamento de Medicina Preventiva e Social da Faculdade de Ciências Médicas da UNICAMP - Campinas, SP - Brasil

Separatas/Reprints: R. Cordeiro - Campus de Botucatu, $\mathrm{s} / \mathrm{n}^{\circ}$ 18618-000 - Botucatu, SP - Brasil

Edição subvencionada pela FAPESP. Processo Medicina 93/ 0208-5. suporte científico para a sua sustentação. Entre eles encontra-se a crença de que se tratam de doenças mais prevalentes nos estratos socioeconomicamente privilegiados; doenças dos países desenvolvidos; doenças fatalmente ligadas ao envelhecimento das pessoas e doenças da urbanização. Certamente tal conjunto de idéias teria grande dificuldade em reproduzir-se se as estatísticas dos países subdesenvolvidos não fossem tão precárias,e se entre os grupos menos privilegiados e nas áreas rurais os serviços de saúde não fossem mais escassos (Costa \& Klein ${ }^{16}, 1985$; Cordeiro ${ }^{14}, 1991$ ). A análise de dados produzidos em países desenvolvidos tem mostrado que a prevalência das doenças cardiovasculares é maior entre as classes trabalhadoras, maior entre os trabalhadores braçais do que entre os administrativos, e que, em pelo menos nos Estados Unidos, Inglaterra, Canadá e Austrália, desde o começo do século as doenças cerebrovasculares vêm declinando,o mesmo acontecendo com as coronarianas a partir dos anos 60 (Gordom \& Thom ${ }^{29}$, 1978; Marmot e col. ${ }^{65}$, 1978; Stallones ${ }^{101}, 1980$; Rose \& Marmot ${ }^{90}$, 1981).Jáhá no Brasil estudos questionando um gradiente crescente de prevalência das doenças cardiovasculares das zonas rurais para as urbanas (Costa $\left.{ }^{15}, 1981\right)$.

A HAS é definida por Stamler ${ }^{102}$ (1970) como uma doença sistêmica caracterizada pela elevação constante da pressão sanguínea, afetando a pressão arterial diastólica e, na maioria das vezes, a pressão arterial sistólica.Entre $120 \mathrm{e} 180 \mathrm{mmHg}$ para a pressão sistólica, e 80 e $110 \mathrm{mmHg}$ para a diastólica, praticamente todos os valores já foram propostos como limites da normalidade da pressão arterial (Pickerning ${ }^{82}, 1972$ ). Em 1978, a Organização Mundial de Saúde estabeleceu como limites para a caracterização da HAS valores maiores ou iguais a 160 e $95 \mathrm{mmHg}$, respectivamente para as pressões sistólica e diastólica (WHO ${ }^{12}, 1978$ ). Na década de 80 várias instituiçðes norte-americanas - particularmente o "National Heart, Lung and Blood Institute" 75,76 (1984, 1985) - constituíram grupos de trabalho com o escopo de padronizar uma definição para a HAS. 
Isto enfatiza a importância e a dificuldade em conceber-se uma definição para a HAS que instrumentalize e operacionalize as ações no sentido de sua prevenção.

\section{Dificuldades na Definiçáo da Hipertensáo Arterial Sistêmica}

A medicina tradicionalmente tem delimitado a HAS através de três aproximações (Rose ${ }^{89}, 1985$ ), que podem ser chamadas de estatística, epidemológica e clínica. A primeira delas, eminentemente estatística, baseia-se na distribuição de frequência das pressões em uma determinada população de interesse, estabelecendo como hipertensos os indivíduos que tiverem suas pressões (sistólica e/ou diastólica) maior do que algo em torno de a média mais dois desvios-padrão.

A segunda aproximação, valorizando aspectos epidemológicos, baseia-se no conceito de risco relativo para definir os limites da HAS. Assim, define como ponto de corte, numa determinada população, valores pressóricos a partir dos quais começa a ser significativo o aumento do risco de aparecerem complicações da HAS. Tal visão da HAS favorece a classificação de diferentes níveis ou graus de hipertensão, uma vez que os riscos de morbidade e mortalidade tendem a crescer com a elevação dos níveis pressóricos, muito embora implique num alto grau de arbitrariedade na definição destes níveis.

Por fim, o terceiro modo de tratar a conceituaçãoda HAS enfatiza os aspectos clínicos ao valorizar a ponderação custo/benefício do tratamento medicamentoso do hipertenso. Isto é, tenta estabelecer como limites da HAS os valores pressóricos a partir dos quais o benefício auferido pelo hipertenso com o tratamento supera os efeitos coleterais das drogas utilizadas. Seguindo este raciocínio, Evans e Rose defíniram as HAS como "um nível de pressão arterial acima da qual o diagnóstico e o tratamento são mais favoráveis do que perigosos"(Evans $\&$ Rose $^{23}$, 1971).

\section{Determinaçáo Social Versus Fatores de Risco}

Mais difícil do que estabelecer limiares para a HAS tem sido, noentanto, compreender a sua etiogênese, particularmente o envolvimento do trabalho em seu aparecimento.

"A pressão arterial elevada é, provavelmente, o mais importante problema de saúde pública nos países desenvolvidos, sendo comum, assintomática, prontamente detectável, em geral facilmente tratável, e muitas vezes letal. se não for tratada." Assim se refere à HAS o livro-texto de clínica médica Harrison Medicina Interna" (Williams \& Braunwald $\left.{ }^{10}, 1984\right)$.

Salientam Williams \& Braunwald ${ }^{110}$ que em 90 a
95\% dos casos de HAS não se consegue elucidar a etiologia da doença. Nenhuma referência é feita à implicação do trabalho na gênese da HAS, exceto por uma menção vaga da importância da ocupação na etiologia da HAS e da implicação da intoxicação pelo chumbo na gênese de HAS de origem neurogênica*.

Em outro livro-texto clássico, "Cecil Textbook of Medicine" (Wingardem \& Smith ${ }^{111}, 1985$ ), nenhuma referência - quer direta, quer indireta - é feita a uma implicação do trabalho na origem da HAS.

Generalizando, pode-se dizer que os livros-texto de clínica médicautilizados noscursos de graduaçãoem medicina no Brasil passam ao largo desta questão.

O mesmo já não se pode dizer a respeito da literatura internacional no campo da saúdeocupacional, onde uma profusão de trabalhos, a partir da década de 60 , tem se voltado para esta questão. Não é intenção proceder aqui a uma revisão exaustiva dessa vasta literatura, nem do ponto de vista dos objetos de estudo, nem do ponto de vista da metodologia empregada. Nesse campo Wang \& Miettinem ${ }^{109}$ (1982) e Harrington ${ }^{34,35}$ (1984), já desenvolveram vários questionamentos relativos ao uso da ocupação enquanto variável independente, à comparabilidade discutível entre as populações estudadas, ao uso de registros pouco confiáveis de exposição e efeitos, entre outros. Ilustra-se no presente artigo apenas os principais aspectos desta discussão.

Uma vez decantada a poeira levantada a partir da célebre polêmica nas décadas de 50 e 60 a respeito da influência dos fatores ligados à herança genética na HAS** $^{*}$ Pickering $^{81}$, 1963; Platt $^{83}$, 1963; Klein \& Araújo $\left.{ }^{52}, 1985\right)$, as atençõesse voltaram para a investigação de fatores ambientais determinados na gênese da hipertensão. Um desses fatores é representado pela ocupação. Esses trabalhos se inserem nos marcos delimitados pela multicausalidade clássica, na linha de investigação epidemiológica proposta, entre outros, por MacMahon $\& \operatorname{Pugh}^{63}$ (1976) e Lilienfeld \& Lilienfeld ${ }^{61}$ (1980).

Os autores que compõem achamadaepidemologia social*** latino-americana criticam esses trabalhos não apenas sob o aspecto metodológico, mas, antes disso, do ponto de vista da filosofia da ciência, enquanto

* Aliás, a HAS secundária à intoxicação pelo chumbo tem origem renal e não nerogênica.

** Como sintetizam Klein \& Araújo ${ }^{52}$ (1985), discutia-se se a HAS seria uma entidade mórbida de caráter qualitativo, Inanifestando-se em pessoas de meia-idade com uma carga genética específica, através de um gen único ou, pelo menos, poucos gens; ou se seria um desvio quantitativo da pressão normal, entendida como uma característica cuja distribuição na população se aproxima da normal (gaussiana), evidenciando assim uma hereditariedade multifatorial, poligênica.

*** Denominaçãoinfelizuma vezque se a epidemiologia estuda a distribuição de características em populações humanas, qual epidemiologia não seria social? 
seus pressupostos gnosiológicos (Breilh $\left.{ }^{7}, 1977\right)$, conforme sintetiza Laurell ${ }^{59}$ (1983): “...Sem dúvida, o modelo multicausal tampouco consegue dar uma resposta satisfatória aos problemas colocados. As razões deste fracasso são de ordem distintas. A mais profunda, talvez, é dada por sua conceituação declaradamente agnóstica, que coloca um paradoxo: pretender explicar partindo da suposição da impossibilidade de conhecer a essência das coisas". No entanto, tal corrente não logrou ainda produzir estudos sistemáticos e em profusão suficiente que permitam concretamente avançar o conhecimento, sob seus pressupostos científicometodológicos, em direção às relações que se estabelessem entre o trabalho e a hipertensão arterial sistêmica, que, aos dias de hoje, aflige mais de 300 milhões de seres humanos*.

Resta, por enquanto, analisar a produçãocientífica existente.

\section{Hipertensão Arterial Sistêmica e Ocupaçăo: Literatura Internacional}

Após estas considerações, - e só após estas considerações - numa visão panorâmica da literatura internacional a respeito da relação entre HAS e ocupação, a observação mais abrangente que se pode fazer é demarcar a tendência à caracterização de uma correlação negativa entre o status ocupacional** e a pressão arterial, quer sistólica. quer diastólica $\left(\right.$ Oakes $^{78}, 1973$; Fouriad $^{24}$, 1984; Opit $^{79}, 1984$ ). Isto é, no mundo do trabalho, as maiores prevalências de HAS são encontradas entre os trabalhadores não especializados, que ganham menores salários, dos setores secundário e terciário da economia (Klein e col. $\left..^{53}, 1986\right)$. Seguindo esta mesma tendência, embora com menor especificidade, alguns trabalhos apontam uma relação inversa entre morbidade e mortalidade por HAS e níveis socioeconômicos, medidos geralmente através da ocupação do indivíduo(McDonoughe col. ${ }^{66}, 1964$; Gordon \& Devine ${ }^{30} .1966$; Skinner e col. ${ }^{100}, 1966$; Dawber e col. ${ }^{19}, 1967$; Metropolitan Life Insurance Company ${ }^{68}$, 1967; Howard \& Holman ${ }^{40}, 1970$; Symi e col. ${ }^{103} .1974$; James \& Kleinbaum ${ }^{+1}$, 1976; HDFP Cooperative Group $^{37}$, 1977; Keil \& Tyroler ${ }^{+9}$, 1977; Marmot e col. ${ }^{65}$, 1978).

Outra tendência observável nos trabalhos internacionais de investigação a respeito das relações entre a HAS e aocupação é a sua fililiação a uma entre duas linhas de pesquisa: a do estresse ocupacional e a dos agentes nocivos físicos e químicos do ambiente.

* Estimativa otimista feita a partir das referências à prevalência da HAS em diversos países e da magnitude população mundial.

** Utiliza-se aqui o sentido que Klein \& Araújos: (1985) deram ao termo.
Não se encontra consenso a respeito da definição de estresse, ou de estresse ocupacional, na literatura. "As definições parecem refletir viéses relacionados à orientação científica dos autores" (Sharit \& Salvendy ${ }^{97}$, 1982). Aliás, antes disso, o que é grave, raramente se encontra uma preocupação em definir-se este conceito. É admirável a imprecisão com que a maioria dos autores trata este termo. Quase invariavelmente os trabalhos se referem a "estresse", "estresse psíquico", "estresse psicossocial" "estresseecológico","estresse ocupacional", sem conceituarem estes termos. Selye ${ }^{95}$ (1976) refere haver mais de 110.000 publicações abordando diferentes aspectos do conceito de estresse no campo da medicina, psicologia e filosofia. Kristensen ${ }^{57}$ (1989), após revisar toda a literatura publicada nos idiomas anglo-saxões e escandinavos entre 1979 e 1988 a respeito da relação entre estresse ocupacional e doenças cardiovasculares, refere que "o campo é caracterizado por uma falta de consenso a respeito do conceito e definição da palavra 'estresse"'. Appley e Trumbul ${ }^{4}$ (1967) afirmam existir "largas variações nos usos específicos, definições específicas e propósitos específicos com os quais o termo estresse tem sido associado". House $^{39}$ (1974) refere que "revisões recentes dos conceitos de estresse social ou psicológico verificaram ser virtualmente impossível definir o que significa estresse a não ser em termos muito gerais". Tais dificuldades em precisar o conceito de estresse têm inclusive levado alguns autores a proporem o seu abandono (Levi ${ }^{60}$. 1971; Kasl $^{18}, 1978$; Mikhail ${ }^{69}, 1981$ ).

Uma rara preocupação em conceituar o estresse ocupacional é percebida em Gardell e col..$^{28}$ (1977), ao abordarem a questão da etiogênese do estresse: "Estudos têm mostrado que a adequada função do sistema nervoso requer um influxo de impulsos do ambiente extemo. Tanto uma sobrecarga quanto uma subestimulação ameaçam o mecanismo homeostático pelo qual o organismo mantém seu adequado nível de vigília. Subestimulação e sobrecarga promovem uma inabilidade dos mecanismos centrais de regulação em manter um nível ótimo de vigília. Este nível ótimo é encontrado em um ponto de equilíbrio de uma escala que vai dos baixos aos altos fluxos de estímulos. ... Quando o fluxo de estímulos é baixo. os indivíduos têm dificuldade em sustentar sua atenção, distraindose e entediando-se facilmente. Do outro lado da escala, o sistema nervoso central é superativado, deteriorando-se a capacidade de concentração. Ansiedade e tensão são desenvolvidas, seguidas de dificuldades em entender e integrar informações. Deteriora-se a capacidade de decisão e ação".

Mais vagamente, House ${ }^{39}$ (1974) assim se refere ao estresse ocupacional: "Estresse é o que ocorre quando as pessoas enfrentam situações onde seu arsenal de comportamentos é insuficiente para sua adaptação. Isto comumente ocorre quando as demandas excedem as habilidades. (...) Insatisfação no trabalho, sobrecarga de 
trabalho ou responsabilidade, conflitos de função e desemprego podem ser consideradas formas de estresse ocupacional."

Para Selye ${ }^{92,93,94}(1956 ; 1973 ; 1975)$, "a essência doestresse é a inespecificidade de um padrão de resposta que pode ser executado por uma ampla variedade de agentes e situações"; para Hinkle ${ }^{38}$ (1974), "A única e essencial característica... nãoé sua falta de especificidade, mas seu alto grau de especificidade".

Alguns autores ainda explicitam ser mais útil falar de "estudo do estresse" como um paradigma do que falar de "estresse" como um conceito. Nesta linha, Karasek $^{45,46,47}(1979 ; 1981 ; 1982)$ construiu um modelo de pesquisa, que leva o seu nome, através de estudos realizados entre as décadas de 70 e 80 , influenciando sobremaneira as pesquisas nesse campo nos anos 80 (Kristensen ${ }^{57}$, 1989). O paradigma de Karasek pressupõe que o estresse ocupacional leva à doença não pela somatória de um conjunto de situações de conflito as mais diversas, mas sim pela relação entre as tensões geradas pelas demandas e exigências do trabalho, por um lado e por outro, pela capacidade que o trabalhador tem de diminuir este nível de tensão, onde particularmente assume papel preponderante a capacidade/possibilidade de tomar decisões proprias no trabalho. $\mathrm{Na}$ visão de Karasek, esses dois aspectos, inerentes ao trabalho, representam respectivamente a fonte de tensão psíquica e seu escoadouro.

Em que pese a imprecisão com que o termo é utilizado, é nítida a tendência em correlacionar níveis altos de pressão arterial e/ou doenças cardiovasculares em geral com estresse ocupacional, quer utilizando o modelo de Karasek (Alfredsson e col. ${ }^{1}$, 1982; Lacroix \& Haynes ${ }^{58}, 1984$; Alfredsson e col. ${ }^{2}, 1985$; Haan $^{32}$, 1985; Theorell e col. ${ }^{107}$, 1987), quer utilizando outras modelagens (Cobb \& Rose ${ }^{13}$, 1973; Theorell e col. ${ }^{106}$, 1977; Theorell \& Floderus-Myrhed ${ }^{105}$, 1977; Zom e col. ${ }^{114}$, 1977; Kornitzer e col. ${ }^{56}$, 1979; Kittel e col. ${ }^{50}$, 1979; Cottington e col. ${ }^{17}$, 1983; Hartvig \& Midttun $^{36}$, 1983; Fouriad e col.24, 1984). Neste último caso, o estresse é responsabilizado como agente causal basicamente através de dois mecanismos: diretamente, pelos mecanismos psicofisiológicos, ou indiretamente, pela indução de comportamentos relacionados ao estresse, tais como o hábito de fumar, o alcoolismo, a hiperalimentação, e outros.

Como um desdobramento das linhas de pesquisa que tentam aprofundar o conhecimento das relações que se estabelecem entre o estresse ocupacional e a HAS, alguns trabalhos têm correlacionado variações humorais de substâncias direta ou indiretamente vasoativas (catecolaminas, corticosteróides, lípides séricos, entre outros) com situações de estresse advindas do trabalho (Frankenhaeuser \& Gardell ${ }^{25}, 1976$; Rahe e col. $^{85}, 1976$ ). Nesta linha, está bem estabelecida a correlação entre o aumento da excreção urinária de catecolaminas e o estresse ocupacional (Astrand e col. ${ }^{6}$,
1973; Rusconi e col. ${ }^{91}, 1975$; Carruthers e col. $.^{10}, 1976$; Timio \& Gentile ${ }^{108}$, 1976; Johansson e col. ${ }^{43}$, 1978; Jenner e col. ${ }^{42}, 1980$ ).

Em síntese, pode-se afirmar que, não obstante as divergências e imprecisões que as idéias de estresse e estresse ocupacional implicam, cada vez mais numerosas têm sido as linhas de pesquisa que convergem para o estabelecimento destas situações como fatores de risco para a HAS.

Uma outra vertente de trabalhos, conforme dito anteriormente, tem buscado estabelecer relações entre fatores físicos equímicos encontrados nos ambientes de trabalhoe HAS. Esses trabalhos consistem basicamente na busca de associações entre riscos físicos ou químicos e HAS, geralmente através de estudos seccionais, e mais raramente através de desenhos longitudinais.

Os achados relacionando exposição ao ruído e pressão arterial são ainda controversos, embora já se possa identificar uma tendência à identificação de associação entre exposiçãoprofissional aruídoe prevalência de HAS. Estando já bem estabelecido o aumento da pressão sistólica como efeito da exposição aguda a alto nivel de pressão sonora (Andren e col. ${ }^{3}, 1982$ ), há trabalhos que encontram associação significativa entre exposição crônica ao ruído e HAS (Parvizpoor ${ }^{80}, 1979$; Jonsson \& Hansson ${ }^{44}, 1977$; Manninen \& Aro $^{64}, 1979$ ), enquanto outros não conseguem identificar esta associação em níveis significativos (Shapiro e col. ${ }^{96}, 1979$ ). Fouriad e col. ${ }^{24}$ (1984) encontraram associação significativa entre exposição profissional e ruídoe prevalência de HAS entre trabalhadores de pequenas e médias empresas de Paris. Talbotte col. ${ }^{104}$ (1985) encontraram, entre trabalhadores de uma metalúrgica portadores de perda auditiva induzida pelo ruído em grau elevado. aumento significativo da prevalência de HAS quando comparados com trabalhadores da mesma fábrica com audição normal. Wu e col. ${ }^{113}$ (1987), em criterioso estudo observacional realizado com trabalhadores de um estaleiro, utilizando modelagens distintas, concluíram que o risco de desenvolver HAS entre expostos cronicamente a altos níveis de ruído, em relação aos não expostos, é cerca de duas vezes maior. Kristensen ${ }^{57}$ (1989), após extensiva revisão a respeito dos efeitos extra-auditivos da exposição ao ruído, refere que, embora seja este ainda um assunto controverso, existem já evidências bastante razoáveis dando suporte à hipótese de associação entre exposição ao ruído e doenças cardiovasculares, afirmando que os trabalhos que não encontramessaassociação são, doponto de vista metodológico. de menor qualidade que os que conseguem firmá-la.

Ainda dentrodesta linha, outros riscosocupacionais têm sido associados à HAS, tais como vibraçōes em baixas frequiências (Gruber \& Ziperman ${ }^{31}$, 1985; Shovkan e col. ${ }^{99}, 1973$ ), exposição ao calor (Kloetzel e col. $^{54}, 1973$; Dukes-Dobos ${ }^{21}$, 1981; Millar, $\left.{ }^{70}, 1988\right)$, exposição crônica a dinitroetilenoglicol (Carmichael \& Lieben $^{8}$, 1963; Einert e col. $.^{22}, 1963$; Mustachi $^{74}, 1977$ ), 
exposiçãoa derivados debenzeno (Reznik \& Vaisman ${ }^{86}$, 1977), exposição ao chumbo (Cramer \& Dahlberg ${ }^{18}$, 1966), exposição ao cadmio (Carrol`, 1966).

\section{Hipertensáo Arterial Sistêmica e Ocupaçăa: Literatura Nacional}

No Brasil ainda são escassas as pesquisas estudando as relações entre ocupação e HAS (Haddad ${ }^{33}$, 1967; Kloetzel e col..$^{54}$, 1973; Klein ${ }^{51}$, 1981; Carvalho e col. ${ }^{12}$, 1983; Ribeiro ${ }^{87}$, 1983; Araújos, 1984; Melhado e col. ${ }^{67}$, 1984; Klein \& Araújo ${ }^{52}$, 1985; Klein e col. ${ }^{53}, 1986$ ). Além disso, exceptuando-se o trabalho realizado na Companhia Siderúrgica Nacional por Klein \& Araújo $^{52}$ (1985), são todos estudos seccionais, onde os dados são colhidos em um só momento da vida da população estudada, o que inviabiliza as já complexas considerações a respeito das relaç̃os causais. Tal limitação metodológica se deve em parte aos custos e complexidade administrativa que os estudos longitudinais envolvem.

A prevalência da HAS entre os grupos profissionais estudados não difere significativamente da encontrada em estudosinternacionais. Melhado e col. ${ }^{67}(1984)$ encontraram $15,6 \%$ e $9,1 \%$, respectivamente para os valores de prevalência da HAS diastólica e sistólica entre opeários da construção civil no Município do Rio de Janeiro em 1984, utilizando-se como limites da normalidade os valores de 90 e $140 \mathrm{mmHg}$.

Klein e Araújo ${ }^{52}$ (1985), em estudo realizado em Volta Redonda, encontraram prevalências de HAS variando entre $2,9 \%$ e $16,0 \%$ dependendo do grupo profissional estudado e após ajustes de idade e outras variáveis.

Carvalhoe col. ${ }^{12}$ (1983) encontraram prevalências entre $6,7 \%$ e $17,6 \%$ para a HAS em diferentes grupos profissionais estudados em algunsmunicípios do Rio de Janeiro, Minas Gerais e São Paulo.

Boa parte desses trabalhos concorda com a tendência internacional acima apontada, encontrando associaçãonegativa entre statusocupacional e prevalência de HAS. Ribeiro ${ }^{87}$ (1983), em estudo seccional realizado na cidade de São Paulo, estudou a distribuição da pressão arterial de mulheres trabalhadoras oriundas de diversos setores da economia, analisando a interferência de algumas variáveis tais como idade, etnia, paridade, migração, hereditariedade, educação, hábito de fumar, uso de anovulatórios orais; bem como algumas variáveis mais diretamente ligadas à ocupação, tais como setor da economia, tipo de trabalho, extensão diária e semanal da jornada. No que diz respeito a estes últimos parâmetros, a pesquisa identificou que a média das pressões diastólicas era significativamente menor entre as trabalhadoras de mais de 35 anos, oriundas de funções administrativas, quando comparadas com um grupo de mesma faixa etária que trabalhava diretamente ligado à produção. E neste último grupo, era significativamente menor entre as trabalhadoras do setor de ensino e têxtil, quando comparada com a média das pressð̃es diastólicas daquelas vindasdo setormetalúrgico e de transporte. Além disso, outro achado no citado trabalho foi a associação entre o número de horas trabalhadas e a pressão diastólica, independentemente deoutras variáveis. Istoé, amédia das pressōes diastólicas das trabalhadoras cujo total de horas trabalhadas por semana era menor que a mediana desta distribuição era menor que a média dos outros $50 \%$ da população. $O$ mesmo acontecia quando se tomava como parâmetro o número diário de horas trabalhadas. A média das pressões diastólicas entre aquelas que trabalhavam até uma hora por dia era menor que a aquelas que trabalhavam entre 1 e 5 horas por dia, que por sua vez era menor que a daquelas que trabalhavam mais do que 5 horas por dia.

Carvalho e col. ${ }^{12}$ (1983), etudando a distribuição da pressão arterial entre homens de 20 a 70 anos, encontraram em valoressignificativos maior prevalência da HAS entre grupos socioprofissionais inferiores, chamando a atenção uma prevalência de HAS maior entre os trabalhadores rurais quando comparada ao trabalhadores urbanos, e maior entre os soldados quando comparadas a militares oficiais.

Klein \& Araújo $^{52}$ (1985) encontraram as médias daspressões sistólicae diastólica, bem comoaprevalência de HAS, sempre crescentes quando se transitava do grupo de ocupações técnicas, científicas e administrativas para o grupo de pequenos burocratas e comerciários; deste para o de operários da indústria de transformação; e finalmente deste para o de trabalhadores dos setores de prestação de serviços, após os devidos ajustes de idade. Paraesses citados autores "existem diferenças significativas entre médias e prevalências dos grupos de ocupação, demonstrandoclaramente que háuma relação entre ocupação e pressãoarterial". Resultados semelhantes já haviam sido encontrados separadamente por Klein ${ }^{51}$ (1981), em trabalho realizado no Rio Grande do Sul, e Araújo5 (1984), no Rio de Janeiro.

\section{Condideraçóes finais}

Pode-se dizer que está bem documentada uma estreita relação entre o trabalho (ou pelo menos a ocupação) e a HAS, cumprindo, pois, explicá-la. Esta difícil tarefa passa necessariamente por um enriquecimento do referencial teórico-metodológico doconjunto das pesquisas em desenvolvimento na área.

A orientação hegemonicamente multicausal impressa aos trabalhos produzidos nos países desenvolvidos merece ser criticada. Sua limitação mais imediata se expressa pela redução que faz de parte considerável e complexa da realidade a uma infinidade de fatores. 
Assim, a prevalência aumentada da HAS entre certos grupos de trabalhadores é entendida como resultante da interação entre vários de eventos e características, tais como o tabagismo (Drettner ${ }^{20}, 1975$ ), a satisfação com o trabalho (Netterstrom \& Juel ${ }^{77}, 1988$ ), a possibilidade de tomar decisões próprias no trabalho (Karasek ${ }^{45}$, 1981), oestresse (House ${ }^{39}, 1974$ ), oestresse ocupacional (Mulders e col. ${ }^{73}, 1982$ ), o estresse social (House ${ }^{39}$, 1974), o estresse ecológico (Mustacchi ${ }^{74}, 1977$ ), a ocupação (French \& Caplan $\left.{ }^{26}, 1970\right)$, o frio (Kristensen ${ }^{57}$, 1989), o calor (Mackie \& O'Hanlon ${ }^{62}, 1977$ ), a filiação voluntária a associações civis (Shekelle e col. ${ }^{98}, 1969$ ), o peso (Talbot e col. ${ }^{104}, 1985$ ), a alimentação (Rusconi e col. ${ }^{91}$, 1975), a exposição ao ruído (Wue col. ${ }^{113}, 1987$ ), a idade (Morris \& Kaagan ${ }^{72}, 1966$ ), o trabalho em turnos (Knutsson $\left.^{55}, 1989\right)$, a cor dos olhos (!) $\left(\right.$ Carter $\left.^{11}, 1980\right)$ e, no limite, tudo o mais que se queira, jogados na vala comum dos fatores de risco* sem uma criteriosa análise qualitativa e, mais importante, ao largo de qualquer consideração que contemple a historicidade do processo que se estuda.

A hegemonia da orientação "multicausalista" impressa aos estudos epidemiológicos tem levado a pesquisa na área a um "beco sem saída". Isto se dá na medida em que a deliberada renúncia à construção de um instrumental teórico capaz de apreender as relações que se estabelecem entre o trabalho e o processo saúde/ doença acaba por forjar um objeto de estudo demasiadamente simplificado.

O desafio - e a responsabilidade - que se coloca aos pesquisadores que têm este entendimentoéo de traduzir a compreensão do processo de trabalho como atividade social em proposições teórico-metodológicas capazes de forjar uma reorientação da pesquisa naárea. Reorientação esta que se contraponha à "naturalização" dos mecanismos que operam a relação entre o trabalho e a HAS. cuja expressão final é a distorção e a compartimentalização do conhecimento, impedindo mesmouma síntese que viabilize uma melhor visibilidade do objeto estudado, além de obstaculizar qualquer aproximação que, transcendendo a aparência, implique em abordagens que cheguem às verdadeiras raízes do processo de adoecimento dos trabalhadores.

CORDEIRO, R. et al. [Work and high blood pressure]. Rev. Saúde Pública, 27:380-7, 1993. Scientific reports on Arterial Blood Hypertension for the period from 1970 to 1989 are reviewed, with special reference to its epidemiological focusing among workers. The knowledge gained and the theoretical and methodological advances associated with it are assessed.

Keywords: Hypertension, epidemiology. Occupations.

* Definidos porKristensen ${ }^{56}$ (1989) como fatores causalmente relacionados às doenças, isto é, fatores cuja presença aumenta o risco de adoecer.

\section{Referências Bibliográficas}

1. ALFREDSSON, L. et al. Myocardial infarction risk and psychosocial work environment: an analisys of the male Swedish working force. Soc.SciMed., 16: 463-7, 1982.

2. ALFREDSSON, L. et al. Type of occupation and near-future hospitalization for myocardial infarction and some ther diagnoses. Int. J. Epidemiol., 14: 378-88, 1985.

3. ANDREN, L. et al. Effect of noises on blood pressure and "stress" hormones. Clin. Sci., 62: 137-41, 1982.

4. APPLEY, M.H. \& TRUMBULL, R. On the concept of psychologicalstress. New York, Appleton-Century-Crofts, 1967.

5. ARAÚJO, J.W.G. Hipertensão arterial em grupos socioeconômicos de Volta Redonda - RJ. Rio de Janeiro, 1984. [Tese de Mestrado Escola Nacional de Saúde PúbliCa/FIOCRUZ].

6. ASTRAND, I. et al. Output and work stress in coastal fishing. Scand.J. Clin. Lab. Invest., 31: 105-13, 1973.

7. BREILH, J. Epidemiologia: economía, medicina y política. Cuidad de México, La Prensa Médica, 1977.

8. CARMICHAEL, P. \& LIEBEN, J. Sudden death in explosives workers. Arch. Environ. Health, 7: 424-33, 1963.

9. CARROL, R.E. The relationship of cadmiun in the air to cardiovascular disease death rates. JAMA, 198: 266-9, 1966.

10. CARRUTHERS, $M$. et al. Man in transit: biochemical and physiological changes during intercontinental flights. Lancet, 1: 967-81, 1976.

11. CARTER, N.L. Eye colour and susceptibility to noise-induced permanent the reshold shift. Audiology, 19: 86-93, 1980

12. CARVALHO, J.J.M. et al. Pressão arterial e grupos sociais. Estudoepidemiológico. Arq.Bras. Cardiol., 40:115-20, 1983.

13. COBB, S. \& ROSE, R.M. Hypertension, peptic ulcer, and diabets in air traffic controllers. $J A M A, 224: 489-92,1973$.

14. CORDEIRO, R. Hipertensão e ocupação: algumas considerações a respeito da epidemiologia da hipertensão arterial sistêmica entre os trabalhadores. Rev. CentroBras. Estudos Saíde, 32: 53-7, 1991.

15. COSTA,E.A. A cross-sectional survey of blood pressure in Rio Grande do Sul - Brazil. London, 1981. [Ph.D Thesis School of Medicine, University of London].

16. COSTA, E.A. \& KLEIN, C.H. Meio urbano e doenças cardiovasculares. Cad. Saúde Pública, 1: 303-9, 1985.

17. COTTINGTON, E. et al. Occupational stress and diastolic blood pressure in a blue-collar population: the Pittsburgh noise-hypertension project. Am.J. Epidemiol., 118: 440-6, 1983.

18. CRAMER, K. \& DAHLBERG, L. Incidence of hypertension among lead workers: a floow-up study based on regular control over 20 years. Br.J. Ind. Med., 23: 101-4, 1966.

19. DAWBER, T. R. et al. Environmental factors in hypentension. In: Stamler, J. et al., eds. The epidemiology of hypertension: proceedings of an international symposium. New York. Grune \& Stratton, 1967. p. 255-82.

20. DRETTENER, B. et al. Cardiovascular risk factors and hearing loss: a study of 1000 fifty-years-old men. Acta Otol., 79 366-71, 1975.

21. DUKES-DOBOS, F.N. Hazards of heat exposure. $J . W k$. Emiron. Health, 7: 73-83, 1981.

22. EINERT, C. et al. Exposure to mixtures of nitroglycerin and ethylene glycol dinitrate.Am.Ind.Hyg.Ass.J.,24:430-40, 1963.

23. EVANS, J.G. \& ROSE, G. Hypertension. Br. Med. Bull., 27: 30-7, 1971.

24. FOURIAD, C. et al. Influence of socioprofessional conditions on blood pressure levels and hypertension control. Am.J. Epidemiol., 120: 72-86, 1984. 
25. FRANKENHAEUSER, M. \& GARDELL, B. Underload and overload in working life. Outline of a multidisciplinary approach. J. Human Stress, 2: 35-46, 1976.

26. FRENCH Jr, J.R.P. \& CAPLAN, R.D. Psychosocial factors in coronary heart disease. Ind. Med., 39: 383-97, 1970.

27. FUNDAÇÃ̂ IBGE. Anutário estatístico do Brasil - 1980. Rio de Janeiro, 1981.

28. GARDELL, B. et al. The working environment for local public transport personel: report. Estocolmo. The Swedish Work Environment Foundation, 1977.

29. GORDOM, R. \& THOM, T. The recent decrease in CHD mortality. Prev. Med., 4: 115-21, 1978.

30. GORDOM, T. \& DEVINE, B. Hypertension and hypertensive heart disease in adults. Vital Health Stat. Ser. 11 (13), 1966.

31. GRUBER, G.J. \& ZIPERMAN, H.H. Apud: Helmkamp, J.C. et al. Whole body vibration: a critical review. Am. Ind. Hyg. Assoc. J., 45: 160-70, 1985.

32. HAAN, M.N.S. Job strain and cardiovascular disease: a tenyear prospective study. Am. J. Epidemiol., 122: 532-9, 1985.

33. HADDAD, N. Inquérito epidemiológico sobre cardiopatias crônicas em um bairro de Ribeirão Preto, São Paulo, Brasil. Arq. Hig. Saúde Pública, $32 / 33$ (111/118): 27-77, $1967 /$ 1968.

34. HARRINGTON, J.M. Occupational mortality. Scand. J. Wk. Environ. Health, 10: 347-52, 1984.

35. HARRINGTON, J.M. Epidemiologic study of work related diseases:methodological problems of register-based studies. Scand.J.Wk. Environ, Health, 10: 353-9, 1984.

36. HARTVIG, P. \& MIDTTUN, O. Coronary heart disease risk factors in bus and truck drivers. Int. Arch. Occup. Environ. Health, 52: 353-60, 1983.

37. HDFP COOPERATIVE GROUP. Race, education and prevalence of hypertension. Am.J. Epidemiol., 106: 351, 1977.

38. HINKLE, L.E. The concept of "stress" in the biological and social sciences. Int. J. Psychiatry Med., 5: 335-57, 1974.

39. HOUSE, J.S. Occupational stress and coronary heart disease: a review and theoretical integration. J. Health Soc. Behav., 15: $12-27,1974$.

40. HOWARD, J. \& HOLMAN, B.L. The effects of race and occupation on hypertension mortality. Milbank Mem. Ftund. $Q ., 48: 263-96,1970$.

41. JAMES, S.A.\& KLEINBAUM, D.G. Socioecologic stress and hypertension related mortality rates in north Carolina. Am. J. Public Health, 66: 354-8, 1976.

42. JENNER, D.A. et al. Catecholamine excretion rates and occupation. Ergonomics, 23; 237-46, 1980.

43. JOHANSSON, G. et al. Psychological and neuroendocrine stress reactions in highly mechanised work. Ergonomics, 21: 583-99, 1978.

44. JONSSON, A. \& HANSSON, L. Prolongued exposure to a stressfull stimulus (noise) as a cause of raised blood pressure in man. Lancet, 1: 86-7, 1977.

45. KARASEK, R.A. Job decision latitude, job demands, and cardiovascular disease: a prospective study of Swedish men. Am. J. Public Health, 71: 694-705, 1981.

46. KARASEK, R.A. et al. Job demands, job decision latitude, and mental strain: implications for a job redesign. Adm. Sci. $Q$., 24: 285-308, 1979.

47. KARASEK, R.A. et al. Job psychological factors and coronary heart disease. Adv. Cardiol., 29: 62-7, 1982.

48. KASL, S.V. Apud: Cooper, C. \& Payner, R. eds. Stress at work. New York, John Wiley \& Sons, 1978. p.3-48.

49. KEIL, J.E. \& TYROLER, H.A. Hypertension: effects of social class and racial admixture. Am.J. Public Health, 67: 6349, 1977.

50. KITTEL, F. et al. Coronary heart disease and job stress in two
Belgian cohorts followed during 10 yr. Eur.J. Cardiol., 9: 455-72, 1979.

51. KLEIN, C.H. Hipertensão arterial em estratos geo-econômicos do Rio Grande do Sul. Rio de Janeiro, 1981. [Dissertação de Mestrado - Escola Nacional de Saúde Pública/FIOCRUZ].

52. KLEIN, C.H. \& ARAÜO, J.W.G. Fumo, bebida alcoólica, migração, instrução, ocupação, agregação familiar e pressão arterial em Volta Redonda, Rio de Janeiro. Cad. Saíde Puiblica, 1: 160-76, 1985.

53. KLEIN, C.H. et al. Variação da pressão arterial em trabalhadores de uma siderúrgica. Cad. Saúde Pública, 2: 212-26. 1986.

54. KLOETZEL, K. et al. Relationship between hypertension and prolongued exposure to heat. J. Occup. Med., 15: 878-85, 1973.

55. KNUTSSON, A. Shiftwork and coronary heart disease. Scand. J. Soc. Med., (Suppl. 44) 1989.

56. KORNITZER, M.D. et al. Incidence of ischemic heart disease in two Belgian cohorts followed during $10 \mathrm{yr}$. Eur. $J$. Cardiol, 9: 455-72, 1972.

57. KRISTENSEN, T.S. Cardiovascular diseases and the work environment: a critical review of the epidemiologic literature on nonchemical factors. Scand.J. Wk. Environ. Health, 15: $165-79,1989$.

58. LACROIX, A. \& HAYNES, S. Occupational exposure to high demand/low control work and coronary heart disease incidence in the Framingham Cohort. Am. J. Epidemiol., 120: $481,1984$.

59. LAURELL, A.C. A saúde como processo social. In: Nunes, E.D., org. Medicina social: aspectos históricos e teóricos. São Paulo, Global, 1983. p. 133-58.

60. LEVI, L. Synopsis of the general discussion. In: Levi, L., ed. Society, stress and disease. London, Oxford University Press, 1971. p.453-76.

61. LILIENFELD, A.M. \& LILIENFELD, D.E. Foundations of epidemiology. New York, Oxford University Press, 1980.

62. MACKIE, R.R. \& O'HANLON, J.F. A study of the combined effects of extended driving and heat stress on driver arousal and performance. In: Mackie, R.R., ed. Vigilance: theory, operational performance and physiological correlates. New York. Human Factors Plenum Press, 1977. (NATO Conference Series III) p. 537-58.

63. MACMAHON, B. \& PUGH, T.F. Principios y métodos de epidemiologia. Ciudad de México, La Prensa Médica Mexicana, 1976.

64. MANNINEN, O.\& ARO, S. Noise-induced hearing loss and blood pressure. Int.Arch.Occup.Environ. Health, 42: 251 6, 1979.

65. MARMOT, M.G. et al. Employment grad and coronary heart disease in British civil sevants. J. Epidemiol. Community Health, 32: 244, 1978.

66. McDONOUGH, J.R. et al. Blood pressure and hypertensive disease in negroes and whites: a study in Evans County, Georgia. Ann.Int.Med., 61: 208-28, 1964.

67. MELHADO, J.C. et al. Avaliação de níveis de pressão arterial em operários da construção civil. Rev.Bras. Saúde Ocup., 12 (45): 67-73, 1984.

68. METROPOLITAN LIFE INSURANCE COMPANY. Cardiac mortality and socieconomic status. Stat. Bull., 48: 9-11, 1967.

69. MIKHAIL, A. Stress: a psychophysiological conception. $J$. Human Stress, 7 (2): 9-15, 1981.

70. MILLAR, J.D. Proposed national strategies for the prevention ofleading work-related diseases and injuries, part I. Am.JInd.Med., 13: 223-40, 1988.

71. MINISTÉRIO DA SAÚDE. Estatisticas de mortalidade, Brasil. Brasília, Centro de Documentação, 1987.

72. MORRIS, J.N. \& KAAGAN, A. Incidence and prediction of 
ischemic heart-disease in London busmen. Lancet, 1:5539, 1966.

73. MULDERS, H.P.G. et al. Differential psychophysiological reactivity of city bus divers. Ergonomics, 25: 1003-11, 1982.

74. MUSTACCHI, $P$. The interface of the work environment and hypertension. Med. Clin. North Am., 61: 531-45, 1977.

75. NATIONAL HEART, LUNG AND BLOOD INSTITUTE. The 1984 report of the joint national committee on detection, evaluation, and treatment of high blood presure. Arch. Intern. Med., 144: 1045-55, 1984.

76. NATIONAL HEART, LUNG AND BLOOD INSTITUTE. National high blood pressure education program working group on risk and high blood pressure: an epidemiological approach to describing risk associated with blood pressure levels. Hypertension, 7: 641, 1985.

77. NETTERSTROM, B. \& JUEL, K. Impact of work-related and psychosocial factors on the development of ischemic heart disease among urban bus drivers in Denmark. Scand J.Wk.Environ. Health, 14: 231-8, 1988.

78. OAKES, T.W.et al. Social factors in newly discovered elevated blood pressure. J. Health Soc. Behav., 14: 198-204, 1973.

79. OPIT, L.J. Occupational and blood pressure. Med J Aust., 140: 760-4, 1984.

80. PARVIZPOOR, D. Noise-induced hearing loss and blood pressure.Int.Arch.Occup Environ.Health, 42:251-6, 1979.

81. PICKERNING, G.W. The inheritance of arterial pressure. In: Pemberton, J., ed. Epidemiology: reports on research and teaching, 1962. London, Oxford University Press, 1963. p. 97-123.

82. PICKERNING, G.W. Hypertension: definitions, natural histories, and consequences. Am.J.Med., 52:570-80, 1972.

83. PLATT, R. Hereditary in hypertension. Lancet, 1: 899-904, 1963.

84. POSSAS, C. A especificidade do quadro sanitário no Brasil: principais características. In: Possas, C. Epidemiologia e sociedade. São Paulo, Hucitec, 1989. p. 19-133.

85. RAHE, R.H. et al. Serum uric acid, cholesterol, and psychological moods thougnout stressfull naval training. Aviat. Space Environ. Med., 47: 883-8, 1976.

86. REZNIK, N.D. \& VAISMAN, V.D. Apud: Mustacchi, P. In the interface of the work environment and hypertension. Med. Clin. North Am. 61: 531-45, 1977.

87. RIBEIRO, M.B.D. Hypertension among female workers in SãoPaulo, Brazil: predictors and joint effects. Hypertension, 5(6): V144-V148, 1983.

88. ROCCELA, E.J.et al. Epidemiologic consideration in defining hypertension. Med.Clin. North Am., 71: 785-90, 1987.

89. ROSE, G.A. Hypertension in the community. In: Bulpitt, C.J. ed.Handbook of hypertension. Amsterdam, ElsevierScience Publishers B.V., 1985, p. 34-49.

90. ROSE, G.A. \& MARMOT, M. Social class and coronary heart disease. Br. Heart J., 45: 13-20, 1981.

91. RUSCONI, C. et al. Fattori di rischio coronarico e cardiopatia ischemica nei conduttori enei bigliettari di autobus. Minervo Cardiol., 23: 718-27, 1975.

92. SELYE, H. The stress of life. New York, McGraw-Hill, 1956.

93. SELYE, H. The evaluation of the stress concept. Am. Scientist, 61: 692-9, 1973
94. SELYE, H. Confusion and controversy in the stress field. $J$. Human Stress, 1: 37-44, 1975.

95. SELYE, H. Forty years of stress research: principal remaaining problems and misconceptions. Can. Med. Assoc. J., 115: 53-6, 1976

96. SHAPIRO, A.P. et al. The role of stress in hypertension. $J$. Human Stress, 5: 7-26, 1979.

97. SHARIT, J. \& SALVENDY, G. Occupational stress: review and reappraisal. Human Factors, 24: 129-62, 1982.

98. SHEKELLE, R.B. et al. Social status incidence of coronary heart disease. J.Chron.Dis. 22: 381-94, 1969.

99. SHOVKAN, N.G. et al. Retinal vessels, intraocular and retinal pressure in patients with vibration disease. Oftal. $Z h ., 28$ : $43,1973$.

100.SKINNER, J.S. et al. Social status physical activity and coronary proneness. J. Chron.Dis., 19: 773-83, 1966.

101.STALLONES, R.A. The rise and the fall of ischemic heart disease. Scient. Am., 243: 43, 1980

102.STAMLER, J. Cardiologia preventiva. Barcelona, Editorial Científico Médico, 1970.

103.SYMI, S.M. et al. Social class and racial differences in blood in blood pressure. Am. J. Public Health, 64: 619-20, 1974.

104.TALBOTT, C. et al. Occupational noise exposure, noise induced hearing loss, and the epidemiology of high blood pressure. Am.J.Epidemiol., 121: 501-4, 1985.

105.THEORELL, T. \& FLODERUS-MYRHED, B. "Workload" and risk of myocardial infarction - a prospective psychosocial analysis. Int. J. Epidemiol., 6: 17-21, 1977.

106. THEORELL, et al. Concrete work and myocardial infarction. Scand. J. Wk. Environm. Health, 3: 144-53, 1977.

107.THEORELL, et al. Psychosocial work conditions before myocardial infarctions in young men. Int.J. Cardiol., 15: $33-46,1987$.

108.TIMIO, M. \& GENTILE, S. Adrenosympathetic overactivity under conditions of work stress. Brit. J.Prev. Soc. Med., 3: 262-5, 1976

109.WANG, J.D. \& MIETTINEM, O.S. Occupational mortality studies: principles of validity.Scand.J.Wk.Environ.Health., 8: $153-8,1982$.

110.WILLIAMS, G.H. \& BRAUNWALD, E. Doença vascular hipertensiva. In:Petersdorf, R.G. Harrison medicina interna. $10^{3}$ ed. Rio de Janeiro, Guanabara Koogan, 1984. p. 1645-59.

111.WINGARDEN, J.B. \& SMITH JR, L.H., eds. Cecil textbook of medicine. 17th ed., Philadelphia, WB Saunders, 1985.

112. WORLD HEALTH ORGANIZATION EXPERT COMMITTEE ON ARTERIAL HYPERTENSION, Geneva, 1978. Report. Geneva, 1978. (WHO Technical Report Series 628).

113.WU, T. et al. Study of noise exposure and high blood pressure in shipyard workers. Am JInd.Med., 12: 431-8, 1987.

114.ZORN, E.W, et al. Ischemic heart disease and work stress in west german sea pilots. J. Occup. Med., 19: 762-5, 1977.

Recebido para publicação em 12.3.1993 Aprovado para publicação em 10.5.1993 